\title{
Study and Design of the Control System for Household Plant Factory
}

\author{
Yanyan Tian \\ Zhengzhou University of Industrial Technology, Henan 451100, China \\ Email: tyy0815@163.com
}

Keywords: Plant factory, ATmega128L, Sensor, LED light source

\begin{abstract}
The crop growth cabinet control system mainly consists of color touch screen interface operating system and control system. They communicate with each other through the RS485 module. The control system's control core is ATmega128L single chip microcomputer, and operating system interface uses Cortex_M3 as the kernel of 32-bit processor STM32F103VC, and adopts $\mu \mathrm{C} / \mathrm{GUI}$ to write human-machine interface which real-time monitors crop growth environmental parameters in the cabinet, and by setting various parameters to simulate the plant a variety of ecological environment.
\end{abstract}

\section{Introduction}

Entering the 21st century, with the development of social economy and the improvement of living quality people begin to have the demand for safe pollution-free vegetables and modern agriculture experience is yearning, and "family plant factory" concept came into being ${ }^{[1]}$. According to the current development trend of the plant factory this paper designs a small plant factory-crop growth cabinet which is suitable for application in the field of family. The plant growth chamber has culture system, sensor data acquisition system, environment control system and control system, etc. and it can simulate the ecological environment for plant growth, thus creating family plant factory.

\section{Composition and Design Scheme of Crop Growth Cabinet}

To realize the function of the cabinet growth system the paper designs the system including hardware and software module. The control system uses RS485 ${ }^{[2]}$ communication technology connect AVR microcontroller and 32-bit processor STM32F103VC processor which the kernel is Cortex_M3 to communicate. AVR microcontroller controls environmental conditions automatically such as plant growth light, temperature, humidity, $\mathrm{CO}_{2}$ concentration, and makes the plants growth inside the facility is not subject to the restriction of natural conditions. As upper machine STM32F103VC processor adjusts and sets light, temperature, humidity, $\mathrm{CO}_{2}$ concentration and other environmental parameters. The system structure diagram is shown in Fig.1.

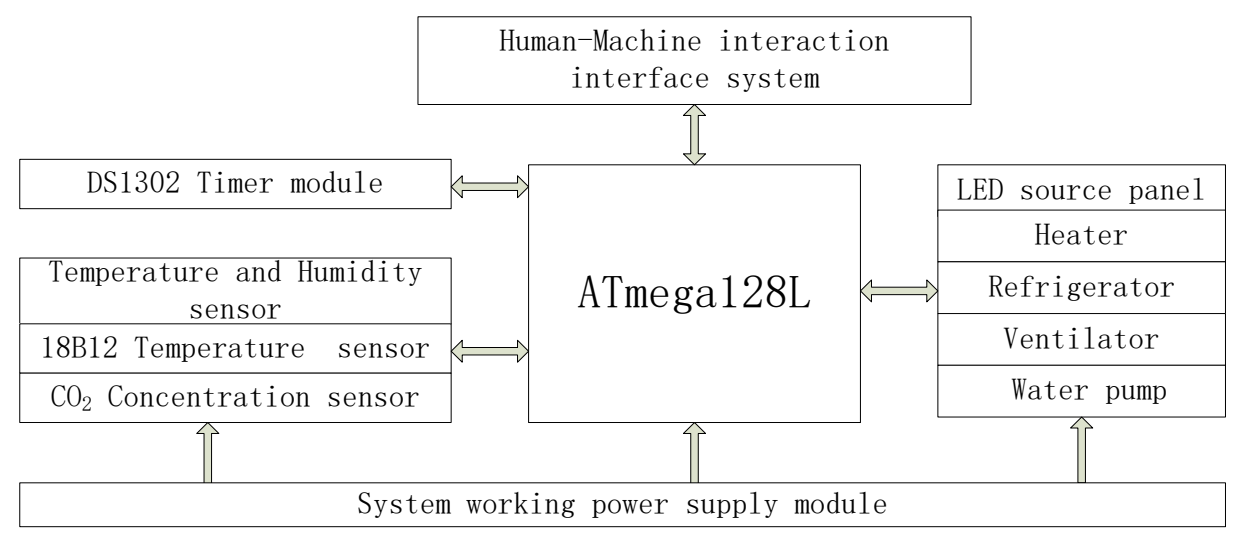

Fig.1 System structure diagram 


\section{System Hardware Design}

\subsection{Power Supply Module.}

The total power of the system has AC220V, DC24V, DC6V and DC5V four kinds. Firstly, the input AC220V converts to DC24V by switching power supply and feeds water pump and LED power supply. Then DC24V converts to DC5V by switching power supply chip LM2576 switching power supply and feeds microcontroller and other accessories. LM2576 can provide 3A output current. Power supply circuit is shown in Fig.2.

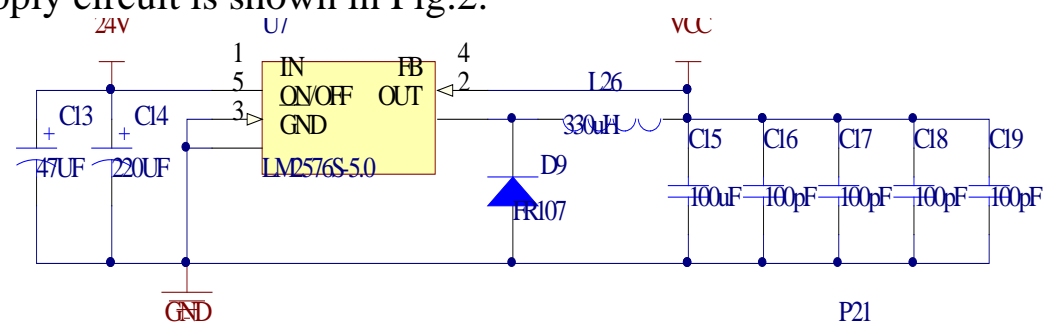

Fig. 2 LM2576 series switching circuit

Due to use 6Vpower supply $\mathrm{CO}_{2}$ module adds a booster module BL8530 that changes DC5V to DC6V and provides to the $\mathrm{CO}_{2}$ module.

\subsection{LED Light Source Module.}

LED light source module adopts LED $^{[3]}$ light panels which is designed by the Chinese Academy of Sciences Institute of Semiconductors. The LED light source plate is composed of multiple type light red and blue LED, and their configuration is in a mixed way.

\subsection{Interface Operating System.}

Interface Operating system adopts 32-bit processor STM32F103VC ${ }^{[4]}$ processor which the kernel is Cortex_M3. The processor is the latest generation of embedded ARM processor and it has advantage of high performance, low power consumption and low cost.

\subsection{Sensor Module.}

In order to give the best environment parameters of different grow period plants needed, and generate a reasonable control scheme, the system configures temperature and humidity sensor, $\mathrm{CO}_{2}$ concentration sensor that the temperature, humidity, $\mathrm{CO}_{2}$ concentration can be collected.

\subsection{1 $\mathrm{CO}_{2}$ Concentration Sensor}

The system adopts solid electrolyte $\mathrm{CO}_{2}$ sensor MG811 ${ }^{[5]}$. The data acquisition of Carbon dioxide concentration is completed by microcontroller ADC converter.

\subsubsection{Temperature and Humidity Sensor}

According to the needs of plant growth in the crop growth cabinet the system uses SHT75 ${ }^{\text {[6] }}$ temperature and humidity sensor. The sensor is a highly integrated temperature and humidity sensor chip.

\section{System Software Design}

\subsection{Control System Program}

The control system program is mainly completed by single chip microcomputer that it can get data acquisition from environment parameter, control external devices and achieve data communication.

\subsubsection{Data acquisition module}

On the basis of the environmental conditions for the growth of plants the module need to collect 
environmental information including temperature, humidity, light and $\mathrm{CO}_{2}$ concentration. Temperature and humidity is acquired by MCU IO mouth directly connected to the temperature and humidity sensors SHT71 interface to complete data collection.

\subsubsection{Peripheral control module}

The external equipments that need to control mainly is including LED light source, the fan, heating Device, refrigerator and water pump. The control signals that single chip microcomputer send are connected to the DC relay of output circuit, and they control the operation of peripherals respectively. The work flow chart is shown in Fig.3.

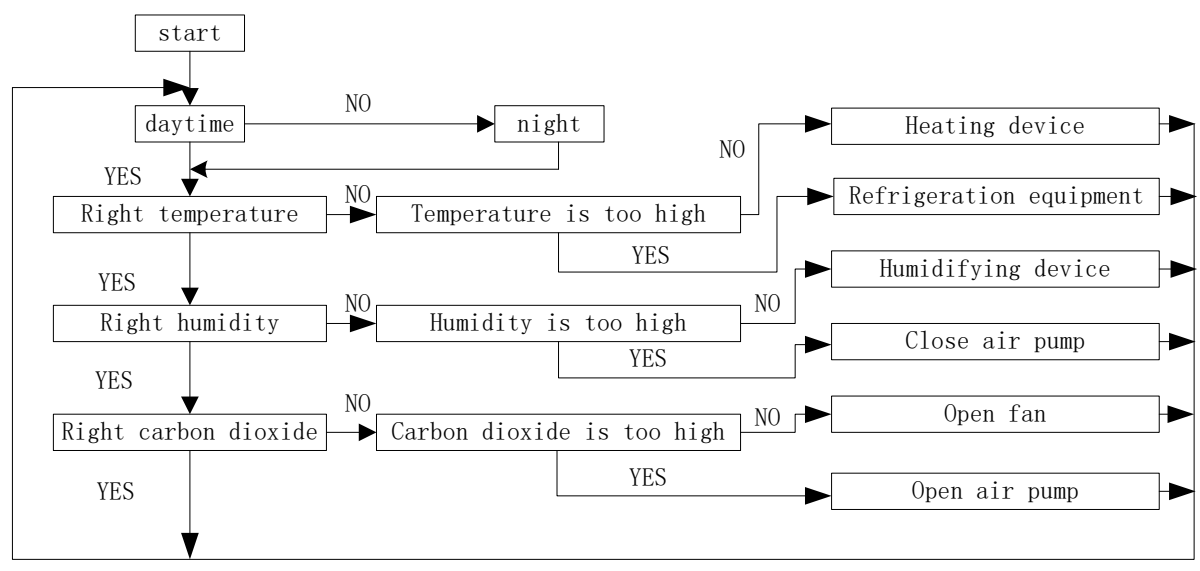

Fig.3 Master control system program flow chart

\subsubsection{Communication module}

Communication module adopts RS485 serial port and the operating systems for data transmission, in order to realize the upload of environment parameters and switch state of the peripheral.

\subsection{Interface Operating Procedures.}

Interface operating procedures to be completed main function manned interaction interface design and data communication with the host system. Human-machine interface is written by $\mu \mathrm{C} /$ GUI. Human-Machine interaction interface uses the menu which has the function of with environmental parameters, mode selection, parameter setting, etc. Its main interface is shown in Fig.4.

\section{System Function Test}

System hardware debugging mainly tests circuit that has been soldered, and software debugging is mainly about liquid crystal display software debugging, and program is written through the ICCAVR software. After testing to verify the system is operating normally, reliable and meets the design requirements.

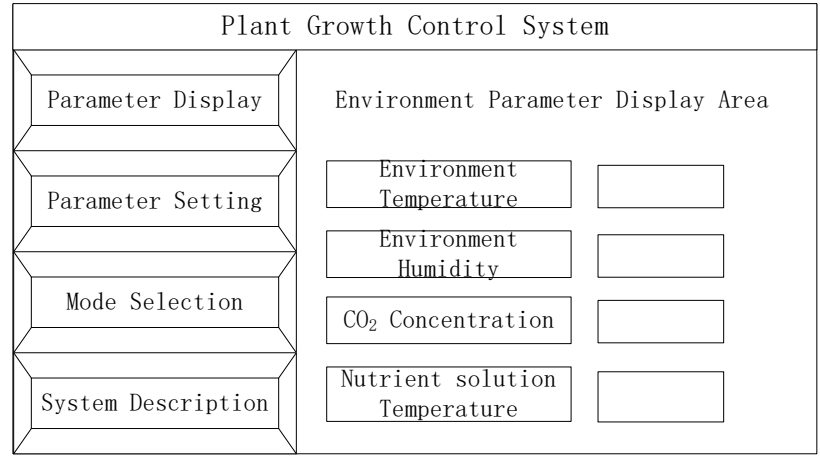

Fig.4 Human-Machine interaction 


\section{Conclusion}

The plant growth environment simulation system this paper designs is based on LED light source is simple and with a relatively low cost, power consumption, meanwhile the control is simple and reliable and better simulate the suitable ecological environment needed for plant growth. It can play a role in the development of plant factory in the future family. After testing to verify the system is operating normally, reliable and meets the design requirements.

\section{References}

[1] Chengbo Zhang, Qichang Yang: Journal of huazhong agricultural university, 34 -37(2004) (In Chinese)

[2] Yanping Xu, Daihua Yang: Journal of electronic science and technology. (02) : 8-10(2009) (In Chinese)

[3] Qichang Yang: Science and technology of China. (01): 52-57(2001) (In Chinese)

[4] Wujie Zhang, Yimin Nan:Journal of computer applications, (10) : 2820-2822(2009) (In Chinese)

[5] Shucheng Chen, Zhiyong Yang and Ke Wang: Journal of microcontroller and embedded systems applications. (01): 47-50(2014) (In Chinese)

[6] Huali Yu, Xiaoshun Zhao and Shuxia Liu: Journal of agricultural mechanization research. (5) :151-153(2008) (In Chinese) 